This document is the accepted manuscript version of the following article: Khaykovich, B., Romaine, S., Ames, A., Bruni, R., Ambaye, H. A., Glavic, A., ... Engelhaupt, D. (2020). Fabrication and testing of high-performance al1-metal neutron guides and axisymmetric mirrors by electrochemical replication. MRS Advances, 5(29-30), 1513-1528. https://doi.org/10.1557/adv.2020.142

\title{
Fabrication and testing of high-performance all-metal neutron guides and axisymmetric mirrors by electrochemical replication
}

\author{
B. Khaykovich ${ }^{1}$, S. Romaine ${ }^{2}$, A. Ames ${ }^{2 *}$, R. Bruni ${ }^{2}$, H. A. Ambaye ${ }^{3}$, A. \\ Glavic $^{3 \dagger}$, V. Lauter ${ }^{3}$, D. Engelhaupt ${ }^{4}$
}

\author{
${ }^{1}$ Nuclear Reactor Laboratory, Massachusetts Institute of Technology, Cambridge, MA \\ 02139, U.S.A. \\ ${ }^{2}$ Center for Astrophysics / Harvard \& Smithsonian, Cambridge, MA 02138, U.S.A. \\ ${ }^{3}$ Neutron Scattering Division, Neutron Sciences Directorate, Oak Ridge National \\ Laboratory, Oak Ridge,TN, U.S.A. \\ ${ }^{4}$ Dawn Research, Huntsville, AL 35824, U.S.A. \\ ${ }^{*}$ Currently at Stanford University, Stanford, CA, U.S.A. \\ + Currently at The Paul Scherrer Institute, Villigen, Switzerland
}

\begin{abstract}
Neutron scattering is one of the most useful methods of studying the structure of matter, with applications to biomedical, structural, magnetic and energyrelated materials. Neutron- scattering instruments are installed around research reactors or accelerator-based neutron sources, and neutron guides are critical components of these facilities. They are neutron- transport optical devices consisting of state-of-the-art mirrors often tens of meters long. Here we demonstrate a novel fabrication method of all-metallic neutron guides and axisymmetric mirrors by electroplating from precision mandrels. The process allows for the fabrication of single-piece all-metal guides of prismatic and axisymmetric shapes. We also demonstrate supermirror guides and axisymmetric focusing supermirrors produced with the same technology. We present the fabrication and tests of the multilayer-coated replicated guides and optic and show that the mandrel is reproduced with high fidelity and reliability. Such supermirror optics will provide game-changing improvements in neutron techniques.
\end{abstract}

\section{Acknowledgements}

This research was supported by the U.S. Department of Energy, Office of Basic Energy Sciences, under Award \# DE-SC0011507 and DE-SC0017098. Research at Oak Ridge National Laboratory's Spallation Neutron Source was sponsored by the Scientific User Facilities Division, Office of Basic Energy Sciences, U.S. Department of Energy. We sincerely appreciate contributions of the late Mikhail V. Gubarev, who is fondly remembered. 


\section{Introduction}

Neutron-scattering techniques require research reactors or accelerator-based installations. These are complex facilities that are normally located at national laboratories, such as Oak Ridge National Laboratory (ORNL) in the US, Institute Laue Langevin in France, J-PARC in Japan, etc. Due to the immense benefits of neutron scattering, such facilities exist around the world and new ones are being constructed or planned. Such facilities consist of a neutron source, surrounded by ten-totwenty experimental end stations, or instruments. Neutrons are delivered to the instruments with the help of neutron guides, long conduits with reflecting surfaces, which often must extend for tens of meters to transport the neutrons from the source to the instrument with the minimum loss to increase the flux illuminating the samples. Therefore, instrument designers invest significant efforts in optimizing the performance of every component of the instruments.

The development of neutron guides is an example of groundbreaking innovation in neutron instrumentation [1]. Before the introduction of guides beam losses between the neutron source and the sample were significant. Guides cut these losses by transporting the neutron beam to the sample position with the help of total external reflection from guide walls. This technology led to an increase in neutron flux by orders of magnitude. Since guides introduction in the 1960s, their quality and design have improved dramatically, such that the guides are now a required component of all neutron-scattering facilities [1]-[3].

The best natural material for reflecting neutrons is nickel (Ni), which is the material of choice for fabricating guides due to its high scattering length density. The critical angle for $\mathrm{Ni}$, below which there will be a total external reflection of the neutrons is the highest among naturally occurring materials. To reduce diffuse scattering and achieve maximum transmission of the neutron beam, the surface quality of guides must be extremely high, with required surface roughness less than or equal to $0.5 \mathrm{~nm}$ (RMS), as measured by AFM.

The expense of fabricating all-nickel (i.e. guides made of nickel only) with such high surface quality has led to the more common fabrication technique of depositing nickel or nickel-titanium multilayers on high-quality flat glass substrates which are then formed into a three-dimensional rectangular shape to produce a guide segment. Glass is the substrate of choice due to its availability and relative ease of polishing to a high surface quality. Float-glass is also used as a substrate and is typically not polished. However, glass is sensitive to harsh environment of irradiation, temperature gradient, and mechanical stress, all of which are especially strong close to neutron moderators. Therefore, glass-based guides are placed not closer than about 1.5 $m$ from moderators [4], [5]. Nickel guides could be placed closer to the 
moderator increasing the throughput ofthe guides.

Because more affordable all-nickel guides would lead to better instrument performance, we have developed and demonstrated an alternative process to optimize andproduce all-nickel neutron guides capable of efficiently delivering neutron beams for tensof meters between neutron moderators and instruments. Our approach to improving guides quality while controlling their rising costs is to adopt the technique ofElectroformed Nickel Replication from precision mandrels [6]-[8].

With this technique, multiple guide sections or individual guide walls can be replicated from a single mandrel (i.e. superpolished glass or metal substrates), as in Figure 1. The resulting guides could either use nickel as the reflecting surface, or they could be coated with multilayers to increase the critical reflection angle. These guides will be especially advantageous when used near neutron sources since metals are more robust than glass in harsh environments near neutron moderators [5].

A mature technology exists to increase the critical angle by producing graded- thickness $\mathrm{Ni} / \mathrm{Ti}$ multilayers, which are called neutron supermirrors [2], [9]. They are made by DC or RF sputtering and may reach thousands of layers, thus the quality of the substrate is paramount. In addition to all-nickel guides, we report the manufacturing and tests of supermirror guides and axisymmetric mirrors. Axisymmetric focusing mirrors have been demonstrated to potentially increase the performance of neutron imaging [10]-[14] and SANS instruments [15], [16]. Coating small radii axisymmetric mirrors with $\mathrm{Ni} / \mathrm{Ti}$ multilayers requires a special process that we have developed and applied here [17], [18]. We achieved a high-quality m-1.9 neutron supermirror on the inner surface ofa 30-mm-diameter conical frustum (45 mm height).

\section{Electroformed Nikel Replication Technique}

In this section, we describe the manufacturing technology of replicated $\mathrm{Ni}$ guides. Two types of mandrels were fabricated for these replication tests: (1) flat mandrels $100 \mathrm{~mm} \times 260 \mathrm{~mm} \times 15 \mathrm{~mm}$ thick and $50 \mathrm{x}$ $260 \mathrm{~mm} \times 15 \mathrm{~mm}$ thick and (2) rectangular 4-sided mandrels $50 \times 100 \times 260$ $\mathrm{mm}$. The flat mandrels were used to fabricate 4 replicas which were then glued together to produce a 3-dimensional guide; therectangular mandrel was used to replicate a 1-piece 4 -sided guide. The mandrels were fabricated from both metal and non-metal. The metal was nickel-phosphorus plated aluminum; the non-metal mandrels included Zerodur and ULE (both ultra-low thermal expansion glasses), silicon and BoroFloat 33 glass.

Electroformed nickel replication creates a replica that faithfully reproduces the figure and surface roughness of a mandrel with high accuracy [19], [20]. X-ray mirrors replicated from metal mandrels have been in use by the Astronomical community for over two decades. They are produced using 
nickel-phosphorus plated aluminum mandrelswhich are single-point diamondturned and polished to a surface finish less than $0.5 \mathrm{~nm}$ (RMS). The sequence of process steps for mandrel preparation and guide fabrication using the electroforming process is described below. It consists of mandrel preparation and certification (or metrology), followed by replication. The mandrels are typically precision machined and polished with a feedback cycle between metrology and polishing. After cleaning and surface passivation, the mandrel is placed into an electrochemical bath for electroforming of metal layers that are later separated from the mandrel by cooling or heating the assembly. The separation of an electroformed part from the mandrel depends primarily on the difference between the coefficient of thermal expansion of the mandrel versus that of the replicated guide and the subsequent difference in temperature from plating process temperature to separation temperature. To reproduce the shape (or figure) of a mandrel with high accuracy the intrinsic stress present in the electroforming process must be minimized. Even small internal stresses intrinsic in the electroformed nickel will aggravate the separation and distort the replica. To control the stress, organic additives containing sulfur and other materials are normally used in the plating solution. These additives are not acceptable for neutron optics since when exposed to a neutron beam they may become activated. Therefore, we developed a method to reduce stress without using additives. A pulsed plating was used where the current is periodically reversed to reduce the stress at the newly grown surface. The current reversal enables the deposition of low-stress nickel while using an extremely low concentration of impurities to avoid excessive activation by the neutron beam. This modified electroformed nickel replication process, which uses forward and reversed rectangular waveforms during the electroforming process was used to fabricate the guides discussed in this paper.

\section{Pulsed Plating}

The methodology we adopted to control the nickel plating stress without using additives was to modify the applied current waveform used to deposit the nickel. Rather than the usual DC current, a pulsed forward and reversed rectangular waveform is used. After significant testing, a process was developed to alter the properties of the deposited nickel, providing the required low stress, suitable uniformity and necessary mechanical properties. In this technique, the plating current is applied in a forward (cathodic) mode at a specific amplitude and then reversed for a significantly shorter time period and higher amplitude (anodic) to remove a minute amount of the deposit. This results in a re- crystallization process which refines the grain size of the deposit. The periodically reversed plating in turn permits additional degrees of freedom in the deposition process which can be selectively adjusted to achieve the most nearly stress-free and uniform deposit. This also provides for a stronger deposit, which in particular, has a higher precision elastic limit that allows for better structural integrity and lower permanent deformation due to stresses applied during assembly and alignment of the overall guide. 
The parameters of the pulsed plating method we developed were determined to minimize the stress during plating. Figure 2 shows the stress vs current density demonstrating the reduction of the stress during pulse plating vs. direct-current plating.

\section{Replicating Guides From Mandrels}

Two different materials have been used to manufacture mandrels, metal and glass. The advantage of the glass is the relative ease of polishing. However, the glass requires heating above boiling-water temperatures to separate replica from mandrels. On the other hand, metal is more difficult to polish but it is easier to separate from the replicaby cooling. Therefore, our developments included the demonstration of both methods of replication.

\section{Metal mandrels}

NASA and the European Space Agency have developed processes to fabricate axisymmetric X-ray focusing mirrors from metal mandrels using DC electroformed nickel replication [21]. To facilitate removal of the electroformed replica from the nickel-phosphorus (NiP) coated Al mandrel after plating, the NiP is first passivated by immersion in dilute potassium dichromate and water solution. A surface reaction of the plating nickel on the passivated mandrel forms a nickel-chromium oxide, which provides the conductive surface required for plating. Since the coefficient of thermal expansion for the aluminum mandrel is higher than for the nickel guide, the $\mathrm{NiP} /$ Aluminum mandrel and electroformed replicas are separated after electroforming via cooling in a chilled water bath. Typically, a DC electroformed replication mandrel is cooled to $\sim 0{ }^{\circ} \mathrm{C}$ from a plating temperature of $\sim 50{ }^{\circ} \mathrm{C}$, producing a difference of about $12 \mathrm{ppm} /{ }^{\circ} \mathrm{C}$. This minute change in size is enough to allow for separation of the replica from the mandrel.

There is a significant difference between axisymmetric mandrels vs. the mandrels for neutron guides. Axisymmetric mandrels typically consist of hyperbolic and parabolic sections [7], [8]. These mandrels have a slight slope from one end to the other which facilitates separation of the optic from the mandrel. Neutron guides are often rectangular parts with straight sidewalls with no slope (draft) from one end to the other, seriously complicating the separation of the guide from the mandrel. If the deposited guide is produced with even small internal tensile stress in the deposit, it will shrink and tightly hug the mandrel when cooled. The minute change in size afforded by the $50{ }^{\circ} \mathrm{C}$ change in temperature requires unreasonably tight specification in flatness or in the slope of the edge corners of the mandrel to prevent the shell locking to the mandrel. To help the release, a taper was added to the corner edges on each mandrel, as shown in Figure 3. Thecorner edges were tapered from $1 \mathrm{~mm}$ deep x $2 \mathrm{~mm}$ wide at one end to $2 \mathrm{~mm}$ deep by $4 \mathrm{~mm}$ wide at the $260 \mathrm{~mm}$ length. This was done at a 45 degree angle and resulted in a flat edge that released the replica easily. We determined that cooling to $-50{ }^{\circ} \mathrm{C}$ is necessary to provide 
adequate clearance to separate the neutron guide from the mandrel. The temperature range of chilled water is too limited to allow separation of the guides from the parallel side rectangular aluminum $/ \mathrm{NiP}$ mandrels. Therefore, a study of available fluids and refrigerants for very low-temperature separation from metal mandrels was undertaken. Upon review of the application temperature ranges and the viscosities at cryogenic temperatures, we chose Fluorinert FC-72. With an application temperature range of $-80^{\circ} \mathrm{C}$ to $70^{\circ} \mathrm{C}$ and a kinematic viscosity of $1.1 \mathrm{~mm}^{2} / \mathrm{s}$ (or cSt) at $-40^{\circ} \mathrm{C}$, this cryogenic lubricating fluid has the desired property of good lubrication at the dry-ice temperatures.

Figure 3 shows an example of a superpolished metal mandrel and resulting nickel guide section separated using this method.

\section{Glass mandrels}

Glass mandrels are very different to manufacture and to use. The coefficient of thermal expansion of the glasses are significantly lower than that of aluminum and nickel discussed above. The Zerodur has nearly zero thermal expansion from sub-zero to well above boiling water temperatures. For separation of the nickel guide from a glass mandrel it is therefore required to heat (rather than cool) the plated mandrel to achieve clearance to remove the guide. Additional issues with the glass include sensitive handling and assembly difficulties required to avoid chipping the glass, especially the edges. For most glass manufacturing processes, special thermal treatment of the material including sharp corners and edges, such as at the chamfers, must be applied in order to anneal the glass. This is required to mitigate stress, especially at cut and ground surfaces. The stress could lead to brittle fracture at reduced impact loads or separation forces resulting in cracking and chipping. Further at issue is the requirement to coat the glass with a thin metallic layer suitable for passivation and separation of the electroformed guide.

The separation media study mentioned above was extended to select a new separation fluid for Zerodur and Silicon low expansion mandrels at above boiling water temperatures. Since the coefficient of thermal expansion of glass is less than that of nickel, plated glass mandrels must be heated to allow for separation of the guide. The plated nickel over glass mandrels must be maintained at elevated temperature consistent with the plating temperature, throughout the plating, rinsing and fixture disassembly. This must then be followed immediately by separation at elevated temperature. (The heated separation method can be dangerous especially if using a fluid such as oil, which may be combustible or may produce explosive vapor.)

During fabrication of initial samples, soybean oil was used for the highertemperature separation of the nickel from glassy material mandrels. This represents a fire hazard since the separation is an open container operation. Therefore, alternative methodsof separation for these processes are desired. The investigation of alternative lubrication fluids (such as $\mathrm{SCH}-60$ ) for immersion separation at high temperatures are being studied. 


\section{Metrology of Mandrels and Replicated Guide Segments}

Great care was taken to measure the mandrels and replicated parts by a number of techniques, including optical profilometry, AFM, x-ray and neutron reflectivity. We tested multiple replicated pieces, both flats and rectangular 3-dimensional guide segments. Bare nickel replicated flats were tested by x-ray reflectometry, AFM, and optical measurements to measure the surface microroughness to compare with the measured surface microroughness of the mandrels. Replicated flats and 3-dimentional rectangular guide segments were also tested at the Magnetism Reflectometer at SNS, Oak Ridge National Laboratory. The parameters such as substrate density, roughness, and multilayer thicknesses have been fitted and compared with optical measurements.

Surface micro-roughness measurements by the manufacturers of the mandrels indicated better surface than our in-house measurements which used different instruments and different filtering and averaging algorithms. For example, for the polished Al mandrel (\#P17-103) the AFM and optical measurements from the manufacturer showed the roughness to be better than 2 $\AA$ (RMS), using low-pass filters and pixel averaging, see Figure 4. We also measured \#P17-103 using a different optical instrument, WYKO, and found that the surface roughness (RMS) was $16 \AA$ without filtering, and $4 \AA$ when low-pass filter was used for data analysis, as shown in Figure 5. The use of different wavelengths for surface measurements may yield different results. As another example, the manufacturer of the ULE glass mandrel flat (\#P16-107) determined a roughness (RMS) of $3 \AA$, while our optical measurements (WYKO) showed roughness between $10-20 \AA$. Nickel replicas from this mandrel showed roughness of $18-25 \AA$ by AFM whichis consistent with the micro-roughness measured for the mandrel indicating that the replication process preserved the surface micro-roughness. Using neutron reflectometry the micro-roughness of this same replica was fitted to $36 \AA$. However, it is difficult to compare the surface roughness from fitted neutron reflectometry data with that from optical measurements, especially for multilayer coated guides. The reflectometry fits are sensitive to the roughness due to the diffraction of neutrons that penetrate much deeper into the surface than optical or AFM probes. Neutrons are also sensitive to changes in density near the surface and the fits are model dependent. The detailed discussion of neutron measurements is below, in the section devoted to neutron reflectometry.

\section{Multilayer Coating}

A dc-magnetron sputtering process was used to coat several flat replicas with supermirrors to increase the critical reflection angle, and hence the throughput of the guides. The base pressure of the chamber was $2 \times 10^{-7}$ Torr; the film was sputtered in Ar at 2.7 mTorr with a target substrate distance of $70 \mathrm{~mm}$. The multilayers were fabricated according to the standard Hayter- 
Mook supermirror recipe [22]. The coatings were fabricated for the m-number $\mathrm{m}=1.9$ and reflectivity $\mathrm{R}=97 \%$. We used $\mathrm{NiC} / \mathrm{Ti}$ multilayers with graded layer thicknesses of approximately: $56 \AA<\mathrm{NiC}<252 \AA$ and $75 \AA<\mathrm{Ti}<135$ $\AA$. All replicas were $1 \mathrm{~mm}$ thick and either $50 \times 100 \mathrm{~mm}^{2}$ or $50 \times 260 \mathrm{~mm}^{2}$. After coating, four replicas were assembled into a guide (using an epoxy glue) with dimensions $50 \mathrm{~mm} \times 100 \mathrm{~mm} \times 260 \mathrm{~mm}$, as shown in Figure 6. The layer sequence specified by the algorithm contained $\mathrm{N}=22$ bilayers.

We have also replicated axisymmetric mirrors with supermirror internal reflecting surface. For this first test we made a conical frustum (a portion of a cone created by slicing the top of a cone by a plane parallel to its base). The inner surface was coated with the same m-1.9 multilayer as the flat guides. In order to coat the inner surface, we have actually coated the mandrel with multilayers. After electroforming the mandrel the multilayers then separate with the replicated mirror [17], [18]. The multilayer-coated mirror was then separated from the mandrel and tested as described below. The Ni/Ti thickness sequence coated on the mandrel was inverted such that the replicated surface had correct supermirror coating.

\section{Neutron Reflectometry of NI and Supermirror Guides}

Neutron reflectivity measurements of replicas and mandrels were performed using the Magnetism Reflectometer at the Spallation Neutron Source at Oak Ridge National Laboratory [23]. The samples were measured in ambient conditions. Measurements of specular reflection were taken over a range of wavelengths from $2.6 \AA$ to $8.6 \AA$, with two bands centred at $6.6 \AA$, and 4.1 A. Figure 7 shows results of the measurements of bare $\mathrm{Ni}$ and multilayer coated Ni guides. The data was fitted using IMD, a standard reflectometry software used for both neutrons and X-rays [24]. Fitting parameters include the density of the substrate and layers, the layer thickness and surface and interfacial roughness.

The surface roughness was also measured by fitting neutron reflectometry data. Several mandrel-replica pairs were compared and it was found that the surface roughness fitting parameters were similar, within a few $\AA$. To further refine the information of the quality of the coating obtained from the neutron reflectometry experiments, we measured the off-specular scattering (OSS) data [25], [26]. The OSS appears due to inhomogeneities in the film and in the interfaces. The off-specular data measured for flat (guide walls) samples are presented in Figure 8. It shows two-dimensional intensity maps as a function of $k_{i z}$ and $k_{f z}$, the projections of the incoming and reflected momenta normal to the surface. Here $Q_{z}$ is the wave-vector transfer of the specular reflection, $k_{i z}+k_{i z}=Q_{z}$, and $k_{i z}-k_{i z}$ is the momentum transfer characterizing the off-specular scattering. The specular reflectivity corresponds to the line at $k_{i z}$ $k_{i z}=0$. The OSS for the Ni coating (Figure 8, top) has features typical for a single film with a rough surface and a single interface with the substrate. In this case OSS intensity develops at the $Q_{z}$ close to the critical $Q_{c}$ at the total reflection region and spreads left and right along $45^{\circ}$ lines with respect to the 
specular reflection. This characteristic OSS channel is the so-called Yoneda scattering [27]. The deposition of the multilayer coating modifies the pattern of the off- specular scattering, shown in Figure 8 (bottom). In addition to the Yoneda wings, a Bragg-sheet scattering develops perpendicular to the specular line. This type of scattering appears from a repeated correlated interfacial roughness (interface to interface) with a distinct contrast between the repeating layers. Our estimate suggests that the corresponding lateral correlations are of $0.2-0.3 \mu \mathrm{m}$.

We examined the Ni replica for the presence of magnetic domains, that might result in a magnetic contribution to the off-specular scattering. For this we measured the reflectivity of one $\mathrm{Ni}$ replica under the applied magnetic field of $1 \mathrm{~T}$ with a polarized neutron beam (polarization $98.5 \%$ within the whole wavelength band) in order to saturate the magnetic domains. The reflectivity curves measured at zero field and above saturation field were identical, thus no magnetization was determined in the film,

Figures 9 and 10 demonstrate neutron reflectivity from the supermirror cone together with a photograph of the cone itself. Figure 9 shows the reflectivity togetherwith the model fit of the multilayer thicknesses and the profile of scattering-length density of the layers. The results indicate that the multilayer was indeed produced according to the recipe. To test the uniformity of the coating over the surface of the cone, a series of measurements have been performed at different azimuthal positions around the circumference. As shown in Figure 9, the reflectivity is identical. The off-specular reflectivity of the cone is shown in Figure 10. It demonstrates features similar to the ones from the flat guide sections giving us the confidence that the axisymmetric supermirrorhas similar reflectivity as from the flat surface.

\section{Summary and Conclusions}

We demonstrated the commercial production of neutron guides by replication from precisely cut and polished mandrels. The production facility has been equipped withelectrochemical baths with controlled chemistry, pulseplating software and hardware, and metrology apparatus and techniques. We demonstrated replication from two mandrel materials: glass (Zerodur, ULE, and borofloat) and metal (Al coated with electroless Ni). Glass mandrels are less expensive to polish than metal ones, and borofloat glass often does not need any polishing. However, replication of prismatic shapes from glass has proved to be more difficult. Glass, especially borofloat glass, tends to chip, damaging mandrels. Besides, the separation of Ni replicas from glass mandrels requires heating to high temperatures (above $100{ }^{\circ} \mathrm{C}$ in oil). Metal mandrels are easier to separate by cooling in ice water or dry ice.

We demonstrated that replicated surfaces have similar roughness and figure (shape) to that of the mandrels of both kinds. Note that the measured roughness depends on the measurement technique and data analysis procedures. 
Therefore, it is important to define not only the microroughness specification but also the method and parameters used for the characterization, as well as any smoothing or averaging software that is used to carry out data analysis.

A major advantage of the electrochemical replication process is that many replicas can be produced from one mandrel, thus long neutron guides that often extend by tens of meters can be uniformly produced from just a few superpolished mandrels. The unique feature of replicated guides is that they can be produced from pure $\mathrm{Ni}$, can be made in almost any prismatic shape without the need for additional assembly, and coated by supermirror multilayers. Such qualities are especially important for guides placed near neutron moderators, where the radiation field is so intense that guides cannot be accessed for service or alignment after being installed.

We also demonstrated the fabrication of replicated multilayer-coated guides and axisymmetric mirrors. The focusing mirrors can produce orders of magnitude higher signal rates in imaging and small-angular scattering applications, potentially enabling revolutionary advances in the performance of neutron instruments [10], [12]-[15], [28], [29]. 


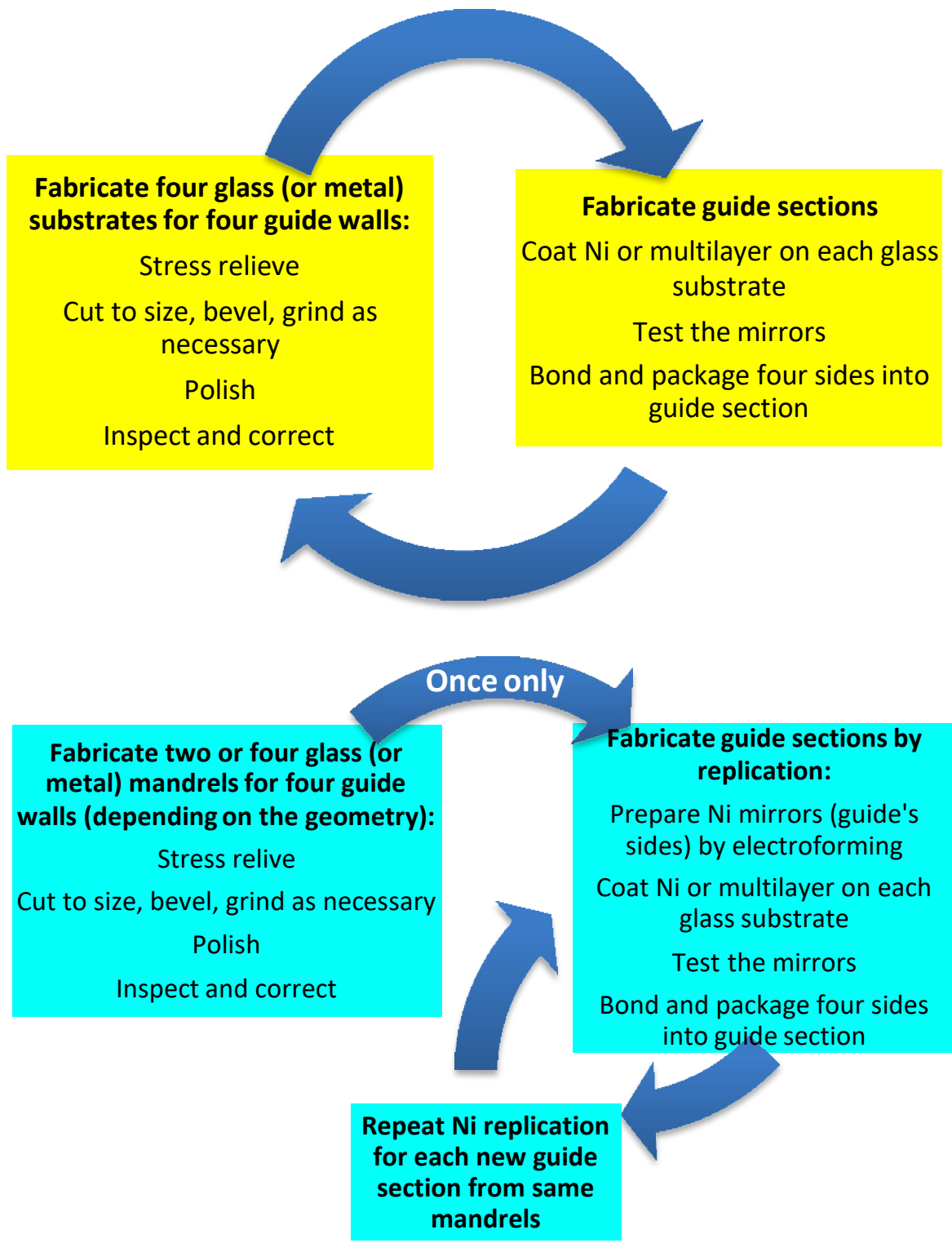

Figure 1. Schematic illustration comparing the existing and the new replication technologies. (Top) Existing method. (Bottom) Our replication method. The main difference is that the replication technology allows for reusing one prepared substrate as a mandrel for replication, rather than preparing multiple substrates for each guide section. 

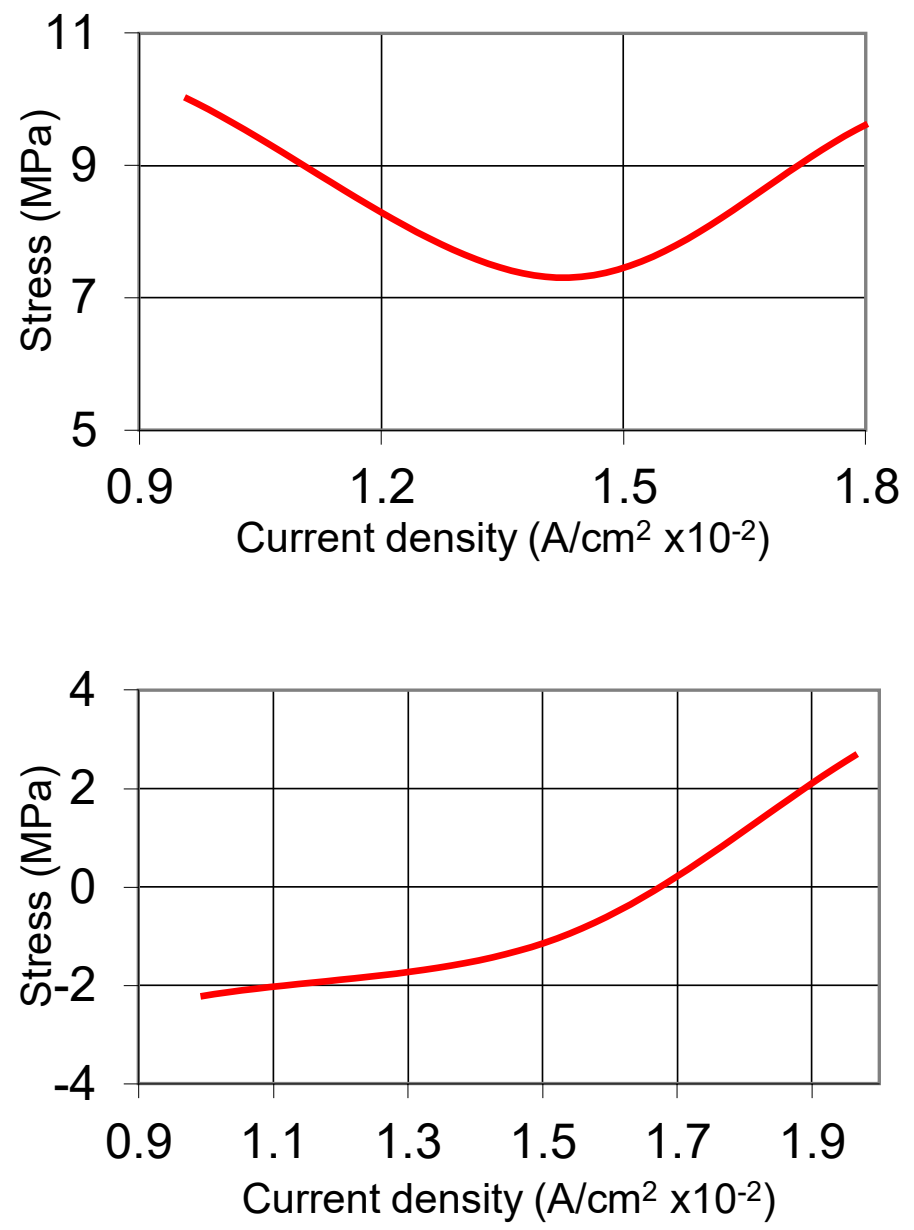

Figure 2. Stress vs. Current Density during plating. (a) DC plating. (b) Pulsed Reverse plating; plating current parameters: forward pulse duration $=18 \mathrm{~ms}$, reverse pulse $=2 \mathrm{~ms}$, ratio of forward to reverse current amplitude is 1:2. Plating temperature $=46^{\circ} \mathrm{C}$. 

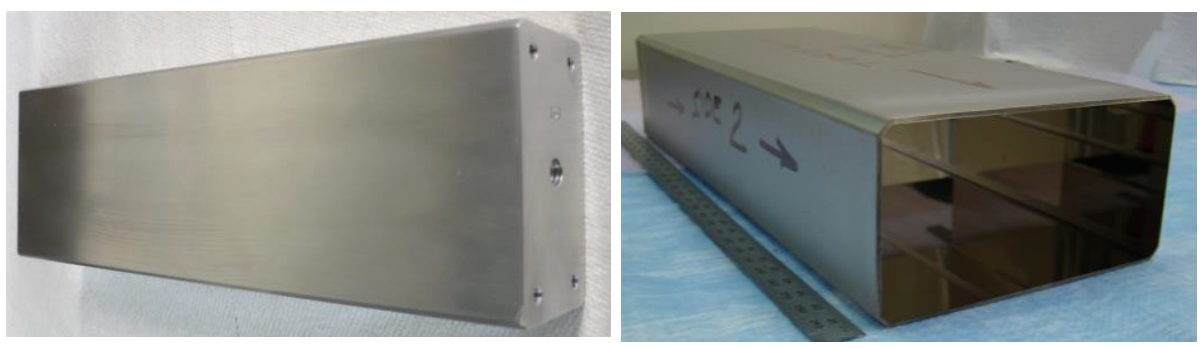

Figure 3. Mandrel (left) and replicated guide (right). Dimensions: $50 \mathrm{~mm}$ x $100 \mathrm{~mm}$ x $260 \mathrm{~mm}$ long. The mandrel has bevelled edges which are tapered along the mandrel to help with the release. The replica was separated from the mandrel using Fluorinert FC-22 container immersed in dry ice (-50 C).

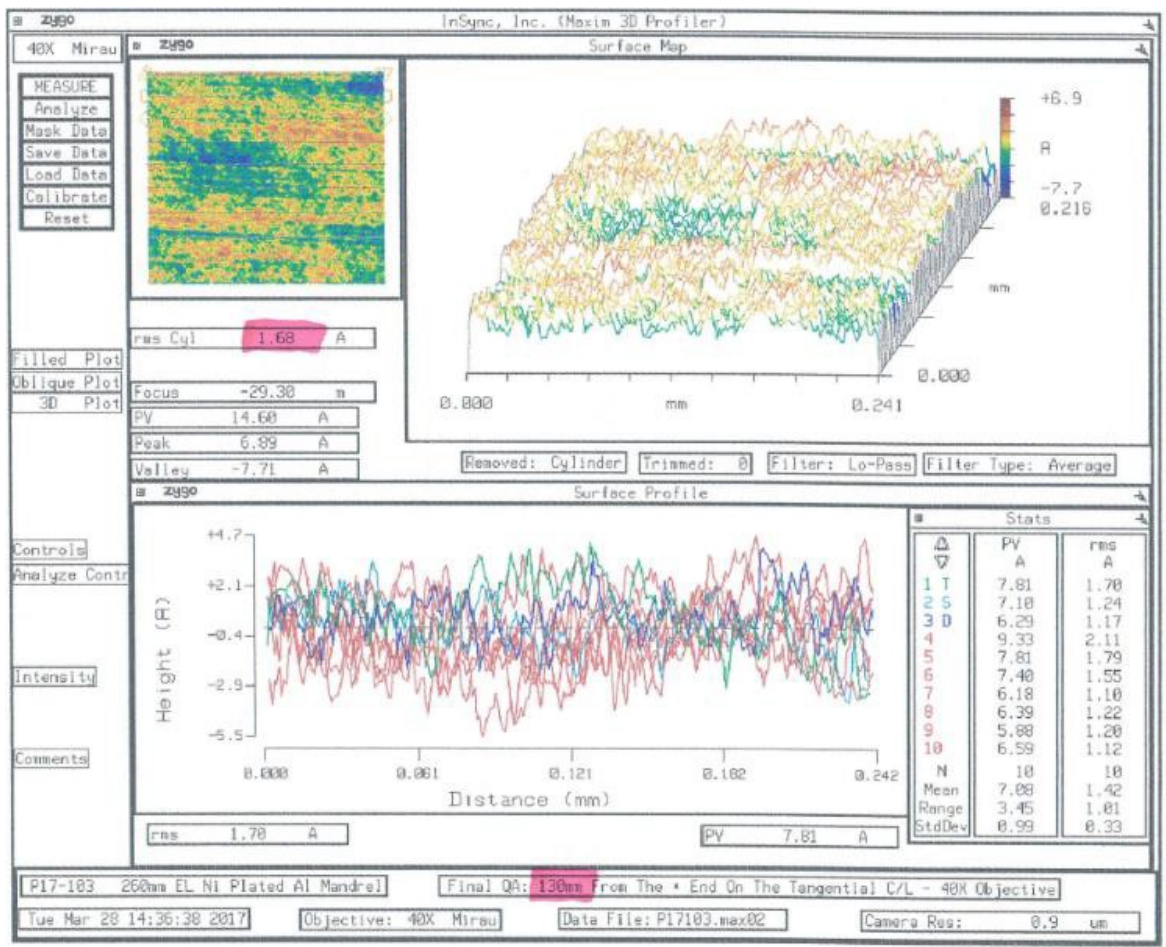

Figure 4. Manufacturer provided optical profilometry (ZYGO) measurements of the polished metal mandrel (P17103). Data is typical of several scans and indicates a roughness (RMS) of the polished metal surface of $\sim 2 \AA$. (lowpass filters and pixel averaging used to process data.) 


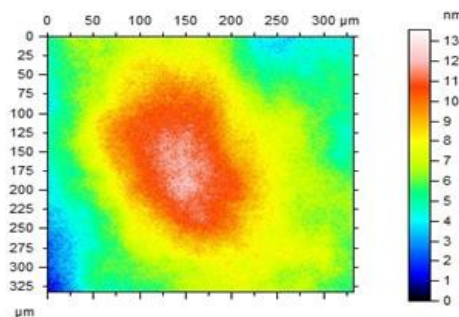

$12^{\text {th }}$ order polynomial removed

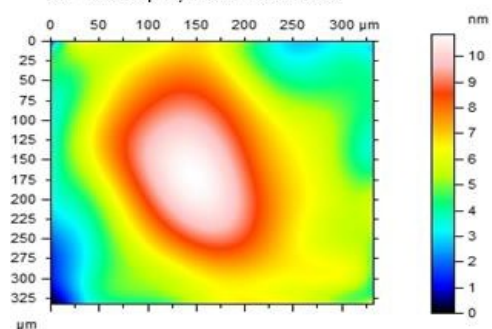

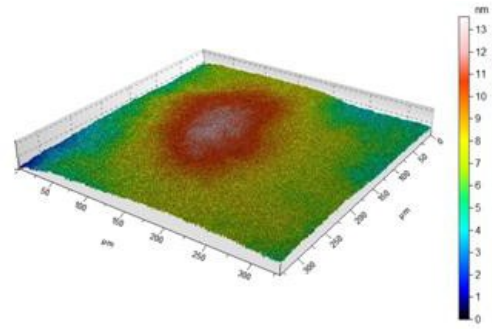

Remaining surface afterterms removal

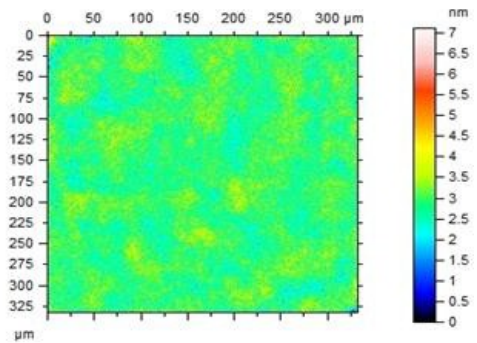

Figure 5. Optical profilometry (WYKO) measurements of the same mandrel as on previous figure. (a,b) show raw data, surface roughness $\sim 16 \AA$; (c) fitted $12^{\text {th }}$ degree polynomial, (d) $12^{\text {th }}$ degree polynomial background removed indicates surface roughness $\sim 4 \AA$. Data is typical of several scans. Data taken by authors. 


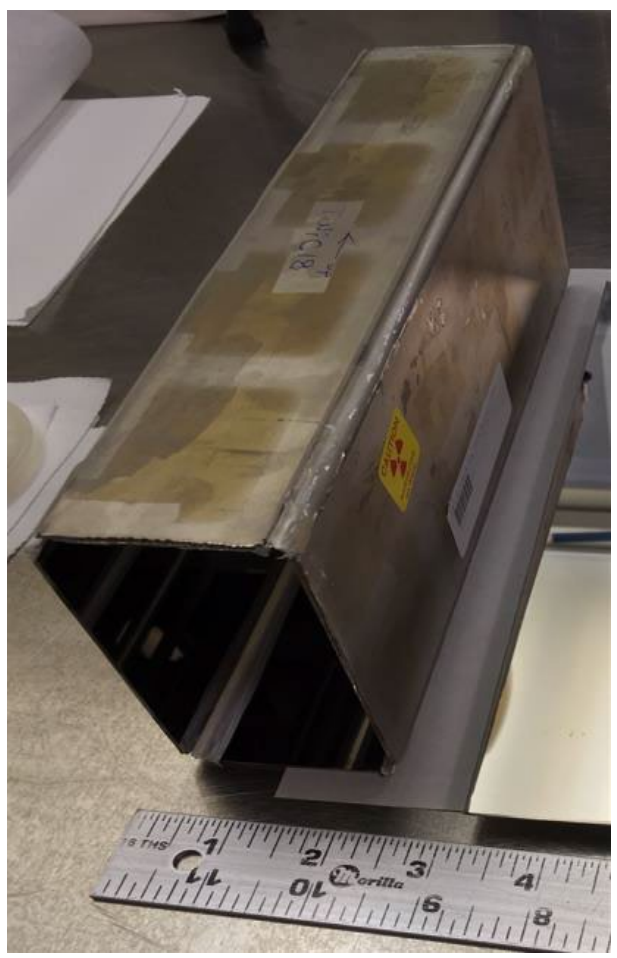

Figure 6. Photograph of a 4-sided multilayer coated guide $50 \mathrm{~mm} \times 100 \mathrm{~mm} \times 260 \mathrm{~mm}$. Four flat replicas were coated with $\mathrm{m}=1.9$ multilayer (see text) and the four sides were then glued together into the guide segment. 

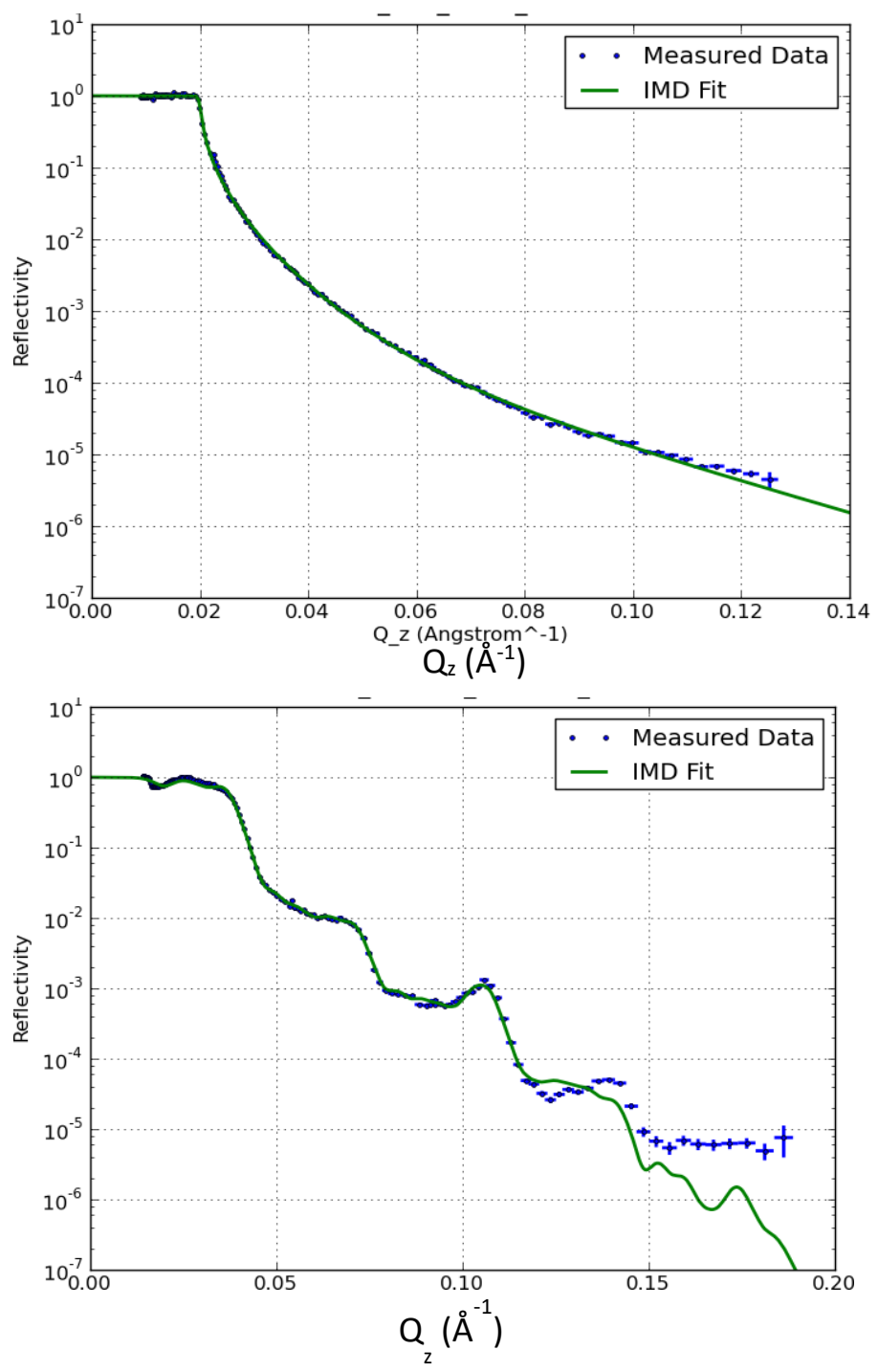

Figure 7. Neutron reflectivity results: plots of specular reflectivity vs. wave vector transfer, $Q_{z}$. Bare Ni guide ( top) and m-1.9 supermirror ( bottom). (Top) The fitting parameters are: NiO layer: $\rho=5.75 \mathrm{~g} / \mathrm{cm}^{3}$ (density), $\mathrm{z}=17.07 \AA$ (thickness), $\sigma=14.23 \AA$ (roughness), Ni substrate: $\rho=8.62 \mathrm{~g} / \mathrm{cm}^{3}, \sigma=22.80 \AA$. ( Bottom) Ti/NiC multilayer. NiC layers: $\rho=5.064 \mathrm{~g} / \mathrm{cm}^{3}$, $\sigma=17.73 \AA, \sigma($ top $)=15.02 \AA, z=58.52-255.73 \AA$, Ti layers: $\rho=3.86 \mathrm{~g} / \mathrm{cm}^{3}, \sigma=16.66 \AA, z=79.95-139.66 \AA$, Ni substrate: $\rho=5.38 \mathrm{~g} / \mathrm{cm}^{3}, \sigma=43.64 \AA$. 


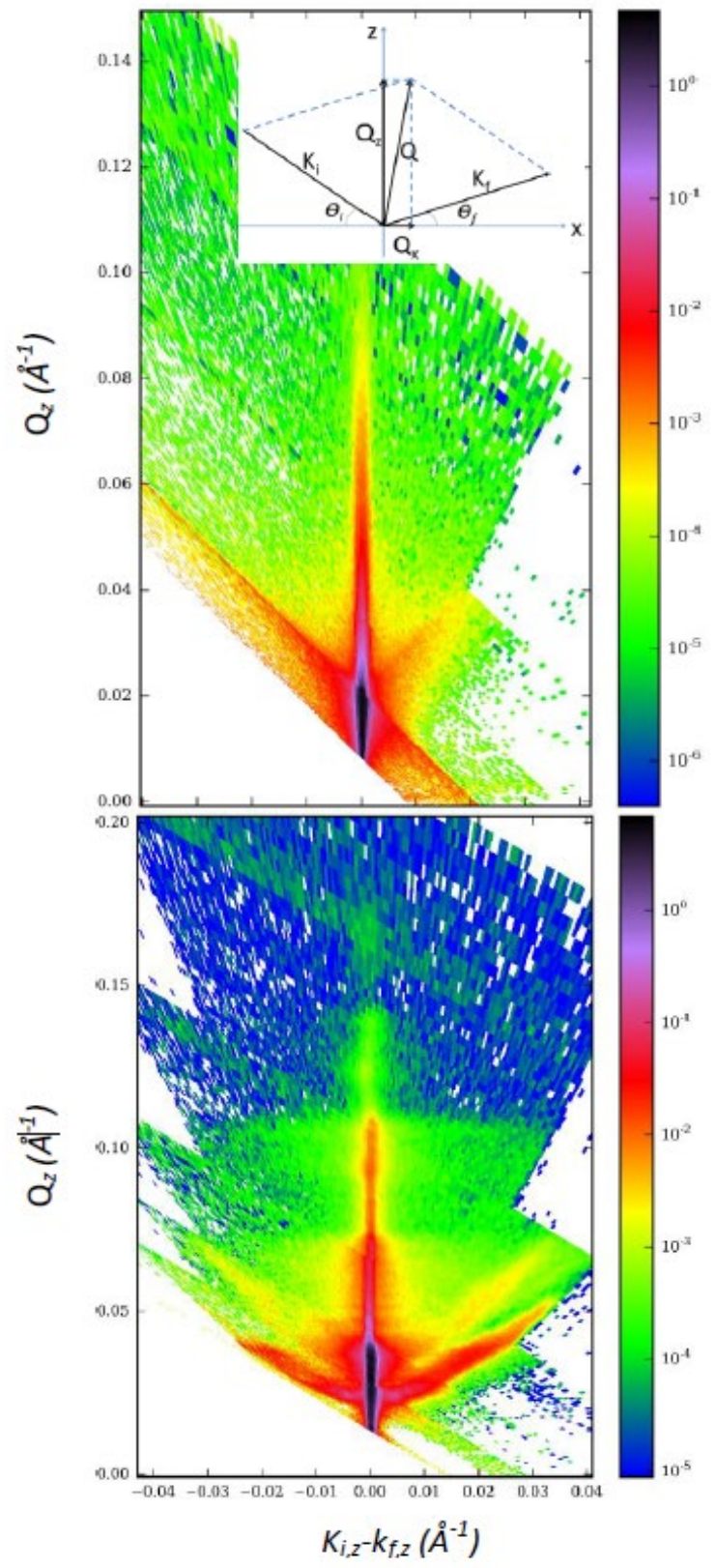

Figure 8. Off-specular scattering pattern. (Top) One side of a 4-sided nickel replicated guide. (Bottom) One side of a 4 -sided $\mathrm{m}=1.9$ supermirror guide section. (Insert) The geometry of the off-specular scattering defining the coordinates $Q_{z}=k_{i z}+k_{i z}$ and $\left(k_{i z}-k_{i z}\right)$. 


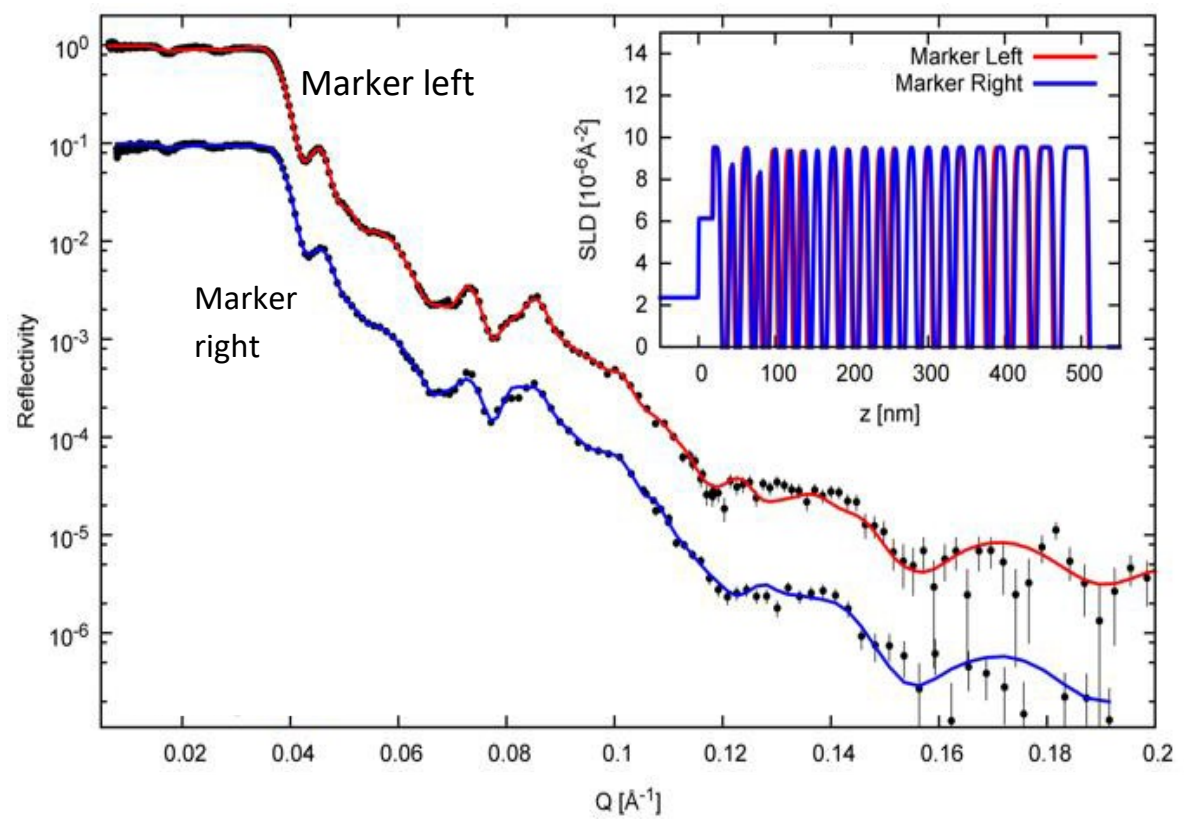

Figure 9. Neutron reflectivity data of multilayer cone $\mathrm{m}=1.9$. The two plots from two different azimuthal scans (marked as "Left" and "Right") show an excellent uniformity of the coating. The data is intentionally shifted for clarity. The inset shows the scattering length density (SLD) profile, obtained after the fit to the data, as a function of the distance from the substrate. The Reflectivity and SLD's are identical for the two azimuthal positions.

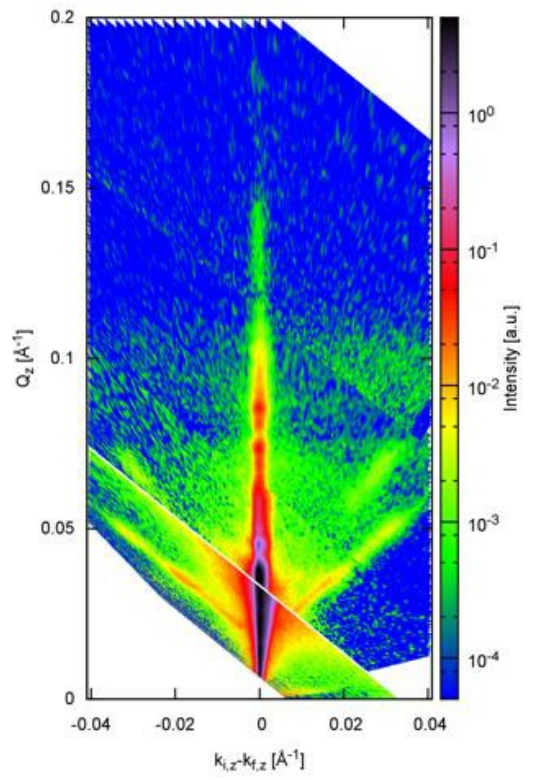

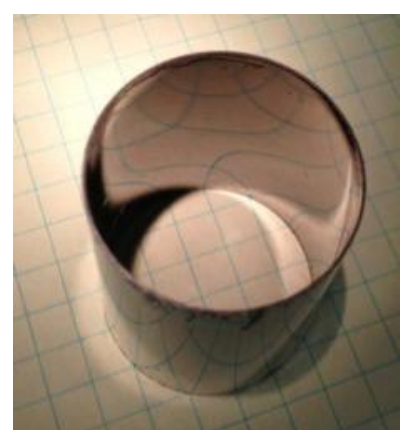

Figure 10. (Left) Off-specular scattering from m-1.9 supermirror conical mirror. (Above) The photograph of the mirror; the conical frustum has an opening angle of about $2^{\circ}$, a height of $45 \mathrm{~mm}$ and a 


\section{References}

[1] H. Maier-Leibnitz and T. Springer, "The use of neutron optical devices on beam-hole experiments on beam-hole experiments," J. Nucl. Energy Parts AB React. Sci. Technol., vol. 17, no. 4, pp. 217-225, Jul. 1963, doi: 10.1016/03683230(63)90022-3.

[2] F. Mezei and P. Dagleish, "Corrigendum and first experimental evidence on neutronsupermirrors," Commun. Phys. Lond., vol. 2, no. 2, pp. 41-43, 1977.

[3] P. Böni, "New concepts for neutron instrumentation," Nucl. Instrum. Methods Phys. Res. Sect. Accel. Spectrometers Detect. Assoc. Equip., vol. 586, no. 1, pp. 1-8, Feb. 2008,doi: 10.1016/j.nima.2007.11.059.

[4] S. Pullen, G. Davidson, S. Pangalis, F. Klose, and S. Kennedy, "Report on the repair of theopal neutron beam transport system," in Joint IGORR 2013 and IAEA TechnologyMeeting, 2013.

[5] C. Schanzer, P. Böni, and M. Schneider, "High Performance Supermirrors on Metallic Substrates," J. Phys. Conf. Ser., vol. 251, p. 012082, Nov. 2010, doi: 10.1088/1742-6596/251/1/012082.

[6] S. C. Fawcett and D. Engelhaupt, "Development of Wolter I x-ray optics by diamond turning and electrochemical replication," Precis. Eng., vol. 17, no. 4, pp. 290-297, Oct. 1995, doi: 10.1016/0141-6359(95)00018-9.

[7] B. D. Ramsey, "Replicated Nickel Optics for the Hard-X-Ray Region," Exp. Astron., vol. 20,no. 1, pp. 85-92, 2005.

[8] B. Khaykovich, M. V. Gubarev, Y. Bagdasarova, B. D. Ramsey, and D. E. Moncton, "From X-ray telescopes to neutron scattering: Using axisymmetric mirrors to focus a neutronbeam," Nucl. Instrum. Methods Phys. Res. Sect. Accel. Spectrometers Detect. Assoc. Equip., vol. 631, no. 1, p. 98, 2011.

[9] F. Mezei, "Novel polarized neutron devices: supermirror and spin component amplifier,"Commun. Phys. Lond., vol. 1, no. 3, pp. 81-85, 1976.

[10] D. Liu et al., "Demonstration of Achromatic Cold-Neutron Microscope Utilizing Axisymmetric Focusing Mirrors," App Phys Lett, vol. 102, p. $183508,2013$.

[11] D. Liu et al., "Response to 'Comment on "Demonstration of achromatic cold-neutron microscope utilizing axisymmetric focusing mirrors"' [Appl. Phys. Lett. 103, 236101(2013)]," Appl. Phys. Lett., vol. 103, no. 23, p. 236102, 2013, doi: $10.1063 / 1.4835175$.

[12] P. Jorba et al., "High-resolution neutron depolarization microscopy of the ferromagnetic transitions in Ni3Al and $\mathrm{HgCr} 2 \mathrm{Se} 4$ under pressure," J. Magn. Magn. Mater., vol. 475,pp. 176-183, Apr. 2019, doi: 10.1016/j.jmmm.2018.11.086.

[13] D. Hussey et al., "Demonstration of Focusing Wolter Mirrors for Neutron Phase and Magnetic Imaging," J. Imaging, vol. 4, no. 3, p. 50, Mar. 2018, doi: $10.3390 /$ jimaging 4030050 .

[14] H. Wu, B. Khaykovich, X. Wang, and D. S. Hussey, "Wolter Mirrors for Neutron Imaging,"Phys. Procedia, vol. 88, pp. 184-189, 2017, doi: 10.1016/j.phpro.2017.06.025.

[15] D. Liu, M. V. Gubarev, G. Resta, B. D. Ramsey, D. E. Moncton, and B. Khaykovich, "Axisymmetric Grazing-Incidence Focusing Optics for Small-Angle NeutronScattering," Nucl Instrum Methods Phys Res A, vol. 686, pp. 145-150, 2012.

[16] D. Liu et al., "Demonstration of a novel focusing small-angle neutron scattering instrument equipped with axisymmetric mirrors," Nat. Commun., vol. 4, p. $2556,2013$.

[17] S. Romaine et al., "Mandrel replication for hard x-ray optics using titanium nitride," Proc. SPIE, vol. 7437, p. 74370Y, 2009.

[18] S. Romaine, J. Boike, R. Bruni, D. Engelhaupt, P. Gorenstein, and B. Ramsey, "Improvedrelease coatings for electroformed x-ray optics," 2011, vol. 8147, pp. 81470W-81470W-6, doi: 10.1117/12.896000.

[19] O. Citterio, P. Conconi, M. Ghigo, F. Mazzoleni, G. Pareschi, and L. Peverini, "Development of soft and hard x-ray optics for astronomy," presented at the International Symposium on Optical Science and Technology, San Diego, CA, USA, 2000, p. 43, doi:10.1117/12.407566.

[20] D. E. Engelhaupt, B. D. Ramsey, S. L. O'Dell, W. D. Jones, and J. K. Russell, "New alloys forelectroformed replicated x-ray optics," presented at the International Symposiumon Optical Science and Technology, San Diego, CA, USA, 2000, p. 154, doi: $10.1117 / 12.407555$.

[21] B. D. Ramsey et al., "The development of hard X-ray optics at MSFC," Proc. SPIE, vol. 
4851,pp. 631-638, 2003.

[22] J. B. Hayter and H. A. Mook, "Discrete thin-film multilayer design for X-ray and neutronsupermirrors," J. Appl. Crystallogr., vol. 22, no. 1, pp. 35-41, Feb. 1989, doi: 10.1107/S0021889888010003.

[23] V. Lauter, H. Ambaye, R. Goyette, W.-T. Hal Lee, and A. Parizzi, "Highlights from the magnetism reflectometer at the SNS," Phys. B Condens. Matter, vol. 404, no. 17, pp.2543-2546, Sep. 2009, doi: 10.1016/j.physb.2009.06.021.

[24] D. L. Windt, "IMD - Software for modeling the optical properties of multilayer films,"Comput. Phys., vol. 12, no. 4, p. 360, 1998, doi: 10.1063/1.168689.

[25] V. Lauter, H. Lauter, A. Glavic, and B. Toperverg, Reference module in materials scienceand materials engineering. Elsevier, 2016.

[26] V. Lauter-Pasyuk, "Neutron grazing incidence techniques for nano-science," Collect. SFN,vol. 7, pp. s221-s240, 2007.

[27] Y. Yoneda, "Anomalous surface reflection of X rays," Phys. Rev., vol. 131, no. 5, p. $2010,1963$.

[28] B. Khaykovich, D. Liu, D. E. Moncton, M. V. Gubarev, and B. D. Ramsey, "A new generation of neutron-focusing optics," SPIE Newsroom, Feb. 2014, doi: $10.1117 / 2.1201401 .005243$.

[29] B. Khaykovich, D. Liu, G. Resta, D. E. Moncton, and M. V. Gubarev, "On the challenge of flux concentration at grazing incidence for neutrons and x-rays," in SPIE Optical Engineering Applications, 2012, vol. 8485, pp. 848509-848509-6. 
